\title{
Ricerche storiche sulla canonistica
}

\author{
A proposito della serie „Der Einfluss der Kanonistik“
}

\section{Intorno a una matrice della cultura giuridica occiden- tale: il diritto canonico medievale (Silvia Di Paolo)}

\begin{abstract}
„Der Einfluss des Kanonischen Rechts auf die europäische Rechtskultur“ costituisce una serie di sei volumi apparsi tra il 2008 e il 2020 nella collana „Norm und Struktur“ (Böhlau) su iniziativa di tre storici del diritto canonico di diversa nazionalità: Orazio Condorelli (Catania), Franck Roumy (Parigi) e Matthias Schmoeckel (Bonn), che hanno promosso un dialogo internazionale intorno al tema del contributo del diritto canonico alla formazione della cultura giuridica europea. Intorno a questa linea di ricerca hanno riunito un nucleo di specialisti provenienti essenzialmente da Italia, Francia e Germania, grazie al sostegno ricevuto dal centro italo-tedesco Villa Vigoni per l'Eccellenza Europea (VV), in collaborazione con la Maison des Sciences de l'Homme (FMSH) e la Deutsche Forschungsgemeinschaft (DFG). Il campo di indagine ha interessato, inizialmente, la storia del diritto e della procedura civile, del diritto pubblico e del diritto penale; in un secondo momento, è stato esteso al diritto processuale, al diritto dell'economia e al diritto internazionale. Ogni ambito disciplinare ha costituito oggetto di una conferenza di ricerca trilaterale, i cui risultati sono stati pubblicati tempestivamente in altrettanti volumi che raccolgono saggi redatti in lingua francese, italiana e tedesca, in nome del plurilinguismo nella ricerca nei campi delle scienze umane e sociali che rappresenta il principio fondamentale del programma concepito dalle tre istituzioni coinvolte.
\end{abstract}

\begin{abstract}
Recensione di: Der Einfluss der Kanonistik auf die europäische Rechtskultur, vol. 1: Zivil- und Zivilprozessrecht, a cura di Orazio Cond orelli, Franck Roumy e Matthias Schmoeckel, XVIII, 445 pp.; vol. 2: Öffentliches Recht, a cura di Franck Roumy, Matthias Schmoeckel e Orazio Condorelli, XII, 447 pp.; vol. 3: Straf- und Strafprozessrecht, a cura di Matthias Schmoeckel, Franck Roumy e Orazio Condorelli, XVIII, 522 pp.; vol. 4: Prozessrecht, a cura di Yves Mausen, Orazio Condorelli, Franck Roumy e Matthias Schmoeckel, XVIII, 361 pp.; vol. 5: Das Recht der Wirtschaft, a cura di David von Mayen burg, Orazio Cond orelli, Matthias Schmoeckel e Franck Roumy, XVI, 488 pp.; vol. 6: Völkerrecht, a cura di Orazio Condorelli, Franck Roumy e Matthias Schmoeckel, XXXII, 392 pp., Wien-Köln-Weimar (Böhlau) 2009, 2011, 2012, 2014, 2016, 2020 (Norm und Struktur. Studien zum sozialen Wandel in Mittelalter und Früher Neuzeit 37/1-6), € 60 (voll. 1, 4), € 65 (voll. 2, 3, 5), $€ 80$ (vol. 6). Gli articoli dei voll. 1-5 sono schedati nel sito di Regesta Imperii: http://www.regesta-imperii.de/; 5.5.2021. Ad oggi manca ancora il vol. 6. - Per la natura del contributo, i riferimenti bibliografici sono limitati ai testi ai quali si fa esplicito rinvio.
\end{abstract}

Kontakt: Silvia Di Paolo, silvia.dipaolo@uniroma3.it; Paola Maffei, maffeipao@unisi.it 
Il titolo della serie ripropone quello di un denso saggio di Peter Landau ( +2019 , alla cui memoria è dedicato il vol. 6), apparso già trenta anni fa, ${ }^{1}$ che riconosce al diritto canonico un rilevantissimo significato rispetto allo sviluppo della cultura giuridica europea, per aver esercitato una specifica influenza sul diritto pubblico, internazionale, penale e privato. Questa prospettiva ha stimolato lo storico del diritto a coltivare una conoscenza più accurata delle fonti del diritto canonico, consentendogli di cogliere meglio il contributo che questo diritto, combinandosi con quello romano, ha dato alla formazione del patrimonio giuridico europeo e, più generalmente, occidentale. Anche grazie all'opera di Francesco Calasso, ${ }^{2}$ che interpretò il diritto comune come un sistema unificatore della tradizione giuridica europea, e poi a quella dei suoi allievi, che hanno ritratto „L'Europa del diritto comune“, ${ }^{3}$ il diritto canonico ha ottenuto sempre maggiore visibilità agli occhi della storiografia giuridica per essere stato uno dei due iura communia vigenti in Europa sino all'età delle codificazioni e per aver esercitato una straordinaria forza moralizzatrice in molti ambiti del diritto in quanto espressione dei valori della società cristiana medievale, oltre che del potere istituzionale della Chiesa. ${ }^{4}$

Le pagine di Landau su „Der Einfluss des kanonischen Rechts auf die europäische Rechtskultur“, che seguono e valorizzano le tracce indelebili lasciate dal diritto canonico sul diritto moderno, hanno trovato ulteriore sviluppo e completamento in un suggestivo lavoro che lo stesso autore dedicò poco dopo al „Bedeutung des kanonischen Rechts für die Entwicklung einheitlicher Rechtsprinzipien“, ${ }^{5}$ che costituì anche il tema di un convegno tenutosi a Berlino nel 1994, a cui parteciparono alcuni tra i maggiori esperti di storia del diritto canonico provenienti da Germania, Francia, Italia e USA: gli stessi hanno contribuito anche ai volumi in oggetto.

1 Peter Landau, Der Einfluss des Kanonischen Rechts auf die europäische Rechtskultur, in: Reiner Schulze (a cura di), Europäische Rechts- und Verfassungsgeschichte. Ergebnisse und Perspektiven der Forschung, Berlin 1991 (Schriften zur Europäischen Rechts- und Verfassungsgeschichte 3), pp. 39-57; ora in: id., Europäische Rechtsgeschichte und kanonisches Recht im Mittelalter. Ausgewählte Aufsätze aus den Jahren 1967 bis 2006 mit Addenda des Autors und Register versehen, Badenweiler 2013, pp. 233-253.

2 Francesco Calasso, Introduzione al diritto comune, Milano 1951; id., Medio Evo del diritto, Milano 1954.

3 Manlio Bellomo, L'Europa del diritto comune. La memoria e la storia, Leonforte 2016.

4 Orazio Condorelli, Il contributo delle ricerche canonistiche alla storia del pensiero medievale, in: Maria Luisa Tacelli/Vincenzo Turchi (a cura di), Studi in onore di Piero Pellegrino, Napoli 2009 (Università del Salento, Collana della Facoltà di Giurisprudenza, n. s. 18), pp. 335-369; id., Il contributo delle ricerche canonistiche alla storia del pensiero medievale: aspetti e problemi, in: Emanuele Conte/Massimo Miglio (a cura di), Il diritto per la storia. Gli studi storico giuridici nella ricerca medievistica, Roma 2010 (Nuovi Studi Storici 83), pp. 65-90, qui p. 82.

5 Peter Landau, Bedeutung des kanonischen Rechts für die Entwicklung einheitlicher Rechtsprinzipien, in: Heinrich Scholler (a cura di), Die Bedeutung des kanonischen Rechts für die Entwicklung einheitlicher Rechtsprinzipien, Baden-Baden 1996 (Arbeiten zur Rechtsvergleichung 177), pp. 23-47; ora in: Landau, Europäische Rechtsgeschichte (vedi nota 1), pp. 255-283. 
Tra questi, Antonio Padoa Schioppa che, in occasione del IX Congresso internazionale di diritto canonico medievale, tenutosi a Monaco di Baviera nel 1992, aveva osservato che se la scienza canonistica giocò un ruolo fondamentale nell'elaborazione delle teorie politiche e giuridiche dell'Occidente, è anche vero che a sua volta essa maturò ,in un rapporto costante di collegamento con altre dimensioni del pensiero e della realtà, collegamento che ... talora è nel senso della derivazione, talaltra in quello dell'influenza“. ${ }^{6}$ Solo un esame puntuale, osservò, avrebbe potuto chiarire come le diverse componenti dello ius commune influirono e furono a loro volta modellate sulla scienza canonistica.

I curatori della serie „Der Einfluss“ hanno portato a piena maturazione le intuizioni e le sollecitazioni provenienti dalla canonistica più sapiente e accorta. Delimitando l'orizzonte di ricerca, potenzialmente molto esteso, entro determinati ambiti del diritto medievale e moderno, anziché entro rigide cornici temporali, essi hanno compiuto un'operazione di restituzione della trama di relazioni e contaminazioni che il diritto canonico ha intrattenuto con le fonti giustinianee, la dottrina civilistica, il pensiero teologico, le svariate normative locali o speciali nonché con le diverse istituzioni, tramite la quale ha contributo in modo essenziale alla formazione della cultura giuridica. Se le modalità e le forme attraverso le quali questo è avvenuto sono state molteplici, a seconda delle circostanze storiche, dei contesti geografici, delle questioni e delle personalità, tuttavia sembra possibile osservare che il diritto canonico, a volte, ha costituito un laboratorio gestazionale di idee e di teorie alle quali gli ordinamenti secolari hanno guardato e attinto per soddisfare finalità temporali, altre volte, ha forgiato meccanismi e procedimenti, ricorrendo a elementi esterni, che hanno funzionato poi da originali modelli operativi. ${ }^{7}$

L'indagine sull'apporto del diritto canonico alla cultura giuridica europea ha valorizzato, esemplificandola, l'attitudine naturale di questo diritto a combinarsi e mescolarsi, in modo sempre originale, creativo e raffinato, con altri elementi della tradizione giuridica, attuando un processo di continua „osmosi“ con elementi esterni al proprio ordinamento e al contempo di rigida „separazione“ fra la sfera delle competenze ecclesiastiche e quella delle competenze secolari, secondo un fenomeno, appunto, di osmosi e di separazione che caratterizza il „modello del diritto canonico“. ${ }^{8}$ A volgere

\footnotetext{
6 Id., Il diritto canonico come scienza nella prospettiva storica. Alcune riflessioni, in: Peter Landau/Joerg Mueller (a cura di), Proceedings of the Ninth International Congress of Medieval Canon Law, Munich, 13-18 July 1992, Città del Vaticano 1997 (Monumenta Iuris Canonici, serie C, Subsidia I), pp. 419-444; anche in: Le ragioni del diritto. Scritti in onore di Luigi Mengoni, Milano 1995, vol. 3, pp. 2045-2077, ora in: id., Studi sul diritto canonico medievale, Spoleto 2017, pp. 251-276, qui p. 274. 7 Antonio Padoa Schioppa (a cura di), Legislation and Justice, New York 1997 (The Origins of the Modern State in Europe, 13th to 18th Centuries 3), p. 342.

8 Id., Réflexions sur le modèle du droit canonique médiéval, in: Revue historique de droit français et étranger 77 (1999), pp. 21-39; in traduzione italiana: Riflessioni sul modello del diritto canonico me-
} 
lo sguardo alle peculiarità di questo diritto accade sempre più spesso che sia anche il giurista europeo più sensibile e accorto, che lo esamina quale ordinamento ,altro“ rispetto allo Stato, in una prospettiva di comparazione con gli ordinamenti civili. ${ }^{9}$ Ad apprezzare la lettura di questa serie di volumi può essere, quindi, lo studioso di cultura giuridica europea, più o meno attrezzato di competenze storiche.

L'uso della categoria „influenza“ ha suscitato, però, qualche ragionevole perplessità. Essa evoca, ha osservato Manlio Bellomo, una condizione di indipendenza e distanza tra due corpi, in ragione della quale uno può esercitare una influenza sull'altro; mentre il legame tra diritto canonico e diritto civile ha costituito „un intreccio e una connessione ineliminabile ... sia quando le normative o le soluzioni dottrinarie convergevano, sia quando divergevano, ma per divergere dovevano tenere conto dell'altro da cui divergevano“. ${ }^{10}$ La ambiguità di questa categoria rischia allora di offuscare la connessione inestricabile tra questi due diritti generali nonché la comune partecipazione alla creazione della cultura giuridica europea, che invece la serie di volumi lascia emergere limpidamente, presentando il diritto canonico come una delle matrici del patrimonio giuridico moderno. Almeno in questa sede, il ricorso alla categoria dell'influsso appare dettato proprio dall'intento di cogliere il contributo effettivo anziché l'influsso - che il diritto canonico, dialogando con le altre componenti del diritto comune, ha offerto al patrimonio giuridico occidentale, per valorizzare l'esperienza europea di ricezione del Corpus Iuris Canonici accanto a quella, comunemente ricordata, del Corpus Iuris Civilis. Questa ratio, che rispecchia e coltiva un valore storico culturale, continua a ispirare altre pregevoli iniziative scientifiche che promuovono la comprensione del comune passato giuridico europeo nella convinzione che „non possa non dirsi cristiano“. ${ }^{11}$

dievale, in: A Ennio Cortese, scritti promossi da Domenico Maffei e raccolti a cura di Italo Birocchi et al., Roma 2001, vol. 3, pp. 21-38; Il modello del diritto canonico, in: id., Italia ed Europa nella storia del diritto, Bologna 2003, pp. 181-208, qui p. 199-204.

9 Maria De Benedetto (a cura di), Il diritto amministrativo tra ordinamenti civili e ordinamento canonico. Prospettive e limiti della comparazione, Torino 2016; ead. (a cura di), Visite canoniche e ispezioni. Un confronto, Torino 2019.

10 Manlio Bellomo, Ius civile, ius canonicum, società medievale, in: Der Einfluss der Kanonistik auf die europäische Rechtskultur, vol. 1: Zivil- und Zivilprozessrecht (vedi sopra), pp. 1-6, qui p. 2.

11 Orazio Condorelli/Rafael Domingo, ,Introduction', in: id. (a cura di), Law and the Christian Tradition in Italy. The Legacy of the Great Jurists, Abingdon-New York 2020 (Law and Religion), pp. 1-24. 


\section{La storiografia canonistica: strade percorse e da percorrere (Paola Maffei)}

Con l'adagio „civilista sine canonista parum, canonista sine civilista nihil valet“, del quale abbiamo notizia almeno dal primo Quattrocento, veniva descritta una situazione di fatto che, in modi e tempi diversi, caratterizzò il cosiddetto sistema del diritto comune nel corso dei numerosi secoli (XII-XVIII) che ne videro la formazione, l'acme e la decadenza; quel sistema - e non il diritto romano tout court, teniamolo bene a mente - che costituisce la vera culla dell'attuale diritto dell'Europa continentale e di quell'amplissima varietà di paesi da essa a vario titolo influenzati.

Oggi, ai fini dello studio delle nostre radici giuridiche, l'adagio prima enunciato potrebbe ben essere capovolto, costituendo lo studio del diritto canonico un'eccellente chiave per apprezzare gli sviluppi e gli esiti del sistema del diritto comune negli attuali paesi a diritto codificato (Europa continentale in primis), nonché nei paesi di common law e perfino nei paesi regolati da sistemi giuridici legati a esperienze statuali teocratiche o marxiste. Dunque, lo studio storico del diritto canonico rappresenta un punto di osservazione del nostro passato giuridico, è insomma uno „speculum iuris historiae“, parafrasando il titolo, „Speculum iuris“, dato da Guglielmo Durante († 1296) al suo trattato di diritto processuale, per alludere alla possibilità di esaminare il diritto sostanziale attraverso il processo.

La serie „Der Einfluss der Kanonistik auf die europäische Rechtskultur“ ben dimostra questo assunto, ed anzi va oltre, esponendo fin dal titolo il suggerimento che il diritto canonico non costituisca solo un mezzo di conoscenza della storia giuridica, ma che esso sia stato uno dei perni fondamentali della formazione di concetti e istituti, apprezzabile in tutte le branche nelle quali è ramificato il diritto.

Ogni volume dei sei che compongono la serie è dedicato ad uno dei numerosi rami nei quali attualmente la scienza giuridica è distribuita: diritto civile e processuale civile, diritto pubblico, diritto e procedura penale, ancora diritto processuale (e ordinamento giudiziario), diritto dell'economia, diritto internazionale. La partizione per materie offre uno dei possibili punti di osservazione, sicuramente il più immediato, della scienza canonistica e della sua presenza nell'evoluzione del diritto. La lettura dei contributi suggerisce inoltre altre prospettive, fra le quali il luogo, il tempo e le fonti dottrinali. Qualsiasi sia la prospettiva di analisi scelta, è possibile stabilire un parallelo fra gli orientamenti della ricerca che emergono dai contributi raccolti in questi volumi e le tendenze generali della storiografia canonistica.

Per quanto riguarda il luogo, premesso che la maggior parte dei saggi è dedicata a sviluppi di concetti e istituti non legati ad un'area determinata, le vie di indagine perseguite dagli studiosi sono prevedibilmente concentrate sull'Europa occidentale continentale; giustamente non trascurate, specie dalla storiografia anglofona, sono le aree di common law; mentre, nel complesso, appaiono sullo sfondo le terre dell'Eu- 
ropa orientale e dell'estremo nord. Le propensioni geografiche sono inevitabilmente dirette verso le terre di più antica cristianizzazione e al contempo maggiormente rigogliose dal punto di vista economico-sociale e più evolute e articolate giuridicamente - come sono quelle „italiane“ e „tedesche“ direttamente soggette al Sacro Romano Impero, quelle „francesi“, nonché la Spagna cristiana - perché forniscono il più ricco, variegato e documentato punto di osservazione. Senza contare che queste terre, e in particolare l'Italia, costituiscono la parte d'Europa dalla quale provengono la maggioranza dei grandi canonisti e da dove ebbe origine e si propagò l'università, istituzione senza frontiere, centro propulsore del sapere e della sostanziale unità giuridica.

Le indagini offerte dai sei volumi rispecchiano peraltro le tendenze geografiche che hanno a lungo dominato la storiografia canonistica, tendenze dovute certamente e prima di tutto ai motivi su esposti, ai quali tuttavia vanno aggiunte quelle cause politiche e religiose che, fino a pochi lustri fa, nell'Europa orientale, baltica e scandinava, hanno costituito un freno ai finanziamenti, alimentando inevitabilmente un certo distacco verso la storia del diritto canonico: infatti nei paesi a fortissima maggioranza protestante la canonistica era interpretata come storia del papato, mentre nei paesi dell'ex blocco sovietico era considerata una forma di fiancheggiamento del sentimento religioso. Per sottolineare il cambiamento di rotta sarebbe interessante promuovere dei colloqui per indagare „den Einfluss der Kanonistik“ nelle varie aree d'Europa e sui diritti particolari (ius proprium) che le caratterizzavano, le cui vestigia sono anch'esse presenti nel mondo giuridico contemporaneo.

Relativamente al tempo, pur non essendo possibile, a causa della estrema varietà dei cento saggi raccolti nei volumi, stilare una vera e propria statistica dei periodi affrontati e fornire numeri precisi, in ogni caso la prospettiva cronologica adottata è delineabile a larghe linee. In via generale, la maggioranza dei contributi segue lo sviluppo di concetti e temi specifici lungo un arco cronologico assai dilatato, comprendente tutto o larga parte del periodo del diritto comune, in vari casi con agganci all'età dei codici: in questi contributi di largo respiro temporale, tuttavia, sembra essere prevalente l'utilizzo delle fonti prodotte nel periodo del cosiddetto diritto canonico classico (dal Decreto di Graziano, 1142-1143, a Giovanni d’Andrea, †1348), mentre per gli altri periodi le ricognizioni sono più sporadiche e concentrate su pochi nomi rappresentativi. Il periodo del diritto canonico classico è inoltre l'oggetto esclusivo di un altrettanto considerevole gruppo di articoli. All'alto medioevo e al periodo pregrazianeo è dedicato un non piccolo insieme di saggi; più o meno altrettanti saggi riguardano il periodo che dal tardo Trecento giunge al Cinquecento. Uno sparuto numero di ricerche si occupa esclusivamente dell'ancien régime.

Gli interessi cronologici manifestati dagli articoli raccolti nei volumi della serie, fortemente sbilanciati verso i circa due secoli che videro lo slancio codificatorio della Chiesa e la redazione delle glosse ordinarie (così chiamate perché accompagnavano indissolubilmente l'ordo, cioè la norma), riflettono l'indirizzo che caratterizza la storiografia canonistica, saldamente orientata verso il cosiddetto diritto canonico classico. 
I motivi di questa preferenza sono molteplici e vari. È sicuramente vero il principio generale per il quale si tende a studiare i fenomeni storici a partire dalle loro prime manifestazioni; e non meno trascurabile è la propensione ad approfondire il momento che di quei fenomeni appare il più creativo. Così è stato non solo per la canonistica ma per la storia del sistema del diritto comune nel suo complesso. Nel caso della canonistica va aggiunta la fortissima, tuttora perdurante influenza degli studi fondamentali di Stephan Kuttner, a partire dall'insuperato „Repertorium der Kanonistik“ (1937). Comprensibilmente Graziano, Gregorio IX e i giuristi di quel periodo esercitano un costante fascino; le ancora incomplete conoscenze sulla realizzazione e diffusione delle loro opere spingono all'approfondimento e ogni nuovo apporto suscita clamore e discussioni, stimolando ulteriori indagini in un circolo virtuoso. Al contrario, dispiace la tutto sommato scarsa attenzione verso un'epoca altrettanto straordinaria come quella che dalla seconda metà del Trecento si spinge fino agli anni della riforma protestante: circa centocinquant'anni di non minore fervore creativo in campo dottrinario e di forte consolidamento in campo normativo e processuale-amministrativo, il cui risultato più appariscente è il testo „definitivo“ del Corpus iuris canonici, rimasto inalterato fino alla fine del 1917. Quanto alla canonistica più tarda, essa sembra suscitare ancor minore interesse, forse perché legata ad una Chiesa che, pur anelando ad una purezza spirituale capace di smentire e superare le critiche che avevano provocato la Riforma, tuttora continua agli occhi di molti ad apparire retriva, decadente e specialmente carnefice nell'uso dell'Inquisizione per perseguire l'ortodossia religiosa e, di riflesso, la fedeltà politica. La lettura dei saggi, unita alla consultazione degli indici che completano ciascun volume, consente infine un'ulteriore prospettiva, quella dei giuristi e delle opere, insomma delle fonti dottrinarie utilizzate dagli autori dei saggi. È ovvio che la preferenza accordata dagli studiosi all'una o all'altra fonte è dipesa innanzi tutto dal tempo e dal luogo preso in esame. Di conseguenza, ampia e capillare attenzione è destinata ai decretisti e decretalisti fino a Giovanni d'Andrea.

Per i saggi dedicati all'epoca successiva - s'intende quella che si spinge agli inizi del Cinquecento - è interessante osservare come da una parte si infittisca la lista dei civilisti vagliati, in particolare dei massimi esponenti, primo fra tutti Bartolo, a riprova che l'utrumque ius costituisce l'impalcatura che sorregge il pensiero dei giuristi di ambedue i diritti e dunque è utile la consultazione non settoriale delle loro opere; dall'altra come l'elenco dei canonisti consultati si impoverisca: i ranghi dei personaggi di primo piano quali a titolo d'esempio Giovanni Calderini, Domenico da San Gimignano, Floriano Sampieri sono assai ridotti. Ovviamente questo è anche il riflesso delle tendenze storiografiche prima accennate della canonistica, che riservano molta minore attenzione ai commentatori rispetto ai glossatori, tanto che mancano studi monografici approfonditi sulla maggior parte dei canonisti tre-quattrocenteschi anche di spicco.

Analoghe considerazioni possono essere fatte per il periodo che dalla Riforma giunge alle porte dell'età delle codificazioni, al quale, come già detto, solo pochissimi autori si sono dedicati in via esclusiva. A parte una meritata attenzione riservata alla 
scuola di Salamanca, l'elenco delle fonti dottrinarie compulsate è ristretto e sembrano essere stati trascurati anche nomi illustri quali il cardinal De Luca. D'altronde anche in questo caso le ricerche raccolte nella serie „Der Einfluss“ sono il riverbero degli orientamenti generali della storiografia canonistica contemporanea, lodevolmente tesa a valorizzare i pensatori spagnoli a lungo trascurati, ma ancora poco incisiva riguardo ai canonisti di quei circa due secoli operanti in altri contesti.

La serie si presenta in una veste grafica elegante con pagine ben leggibili. In ciascun volume la premessa in tre lingue - tedesco, francese e italiano - offre un panorama non superficiale dei cento saggi presentati: 18 nel primo e nel quinto, 17 nel secondo e nel terzo, 16 nel quarto, 15 nel sesto, per mano di 41 autori. I volumi, completati da tre utilissimi indici (rerum, personarum, canonum et legum), sono ingentiliti da una simpatica fotografia dei partecipanti ai colloqui e (salvo il terzo) da uno schizzo del luogo dell'incontro di Hans-Georg Hermann. Nel complesso questa serie fornisce un importante tassello alla conoscenza del fondamentale contributo dato dal diritto canonico allo sviluppo del diritto europeo e per conseguenza alla formazione della cultura giuridica di larga parte del mondo odierno. 